Federal Reserve Bank of Minneapolis Research Department

\title{
Stochastic Optimal Growth with a Non-Compact State Space*
}

Yuzhe Zhang

Working Paper 639

October 2005

\begin{abstract}
This paper studies the stability of a stochastic optimal growth economy introduced by Brock and Mirman [J. Econ. Theory 4 (1972)] by utilizing stochastic monotonicity in a dynamic system. The construction of two boundary distributions leads to a new method of studying systems with noncompact state space. The paper shows the existence of a unique invariant distribution. It also shows the equivalence between the stability and the uniqueness of the invariant distribution in this dynamic system.
\end{abstract}

Keywords: stochastic growth, stochastic dominance, monotonic operator, global stability

JEL Classification: C61, C62, O41

*Zhang, University of Minnesota and Federal Reserve Bank of Minneapolis. The views expressed herein are those of the author and not necessarily those of the Federal Reserve Bank of Minneapolis or the Federal Reserve System. 


\section{Introduction}

As one of the most useful workhorses in modern economics, the stability properties of the stochastic growth model have been extensively studied. The original work on stochastic growth was by Brock and Mirman (1972). They extended the deterministic growth model of Ramsey (1928), Cass (1965), Koopmans (1965) and others to a stochastic setting. They showed that the existence, uniqueness and stability results of the deterministic case are also realized under similar assumptions on preference and production technology. In their analysis, certain restrictions were imposed on the production function and distribution of shocks. In particular, the shock was assumed to have a compact support and this helped to provide a compact state space in their analysis. Subsequent research has aimed at relaxing some rigid assumptions of Brock and Mirman's work. Stachurski (2002) studied a stochastic model in which the shock was unbounded. His paper was based on recent innovations in the theory of stochastically perturbed dynamic systems. In his paper, the state of the economic system (i.e. distribution of output at the beginning of time $t$ ) was represented by a density. He showed that the system is both Lagrange stable and strongly contractive, which are sufficient conditions for stability.

Nishimura and Stachurski (2005) used results from irreducible Markov chain theory to study stability. Their paper proposed a Euler equation technique for analyzing the dynamic system. It combined the Euler equation of the optimal program with the Foster-Lyapunov theory of Markov chains. The simplicity of this technique allows the elimination of several conditions required in earlier studies.

Nishimura and Stachurski's results are based on the irreducibility ${ }^{1}$ of the Markov chain. In order to guarantee that, they assumed that the productivity shock has a density and that the density is positive everywhere. While this assumption is innocuous in their context, irreducibility is either too restrictive or simply very difficult to verify in many other environments. This is particularly the case in economic models with state variables including both exogenous and endogenous variables. To fix these ideas, let us examine a simple example. Suppose that in a dynamic economic model there are exogenous shocks

\footnotetext{
${ }^{1} \mathrm{~A}$ Markov process with state space $S$ and transition kernel $Q(\cdot, \cdot)$ is called irreducible if there is a non-zero measure $\psi$ on $S$ such that for any initial point $s \in S$, and any subset $B$ with positive measure under $\psi, \operatorname{Pr}\left\{s_{t} \in B \mid s_{0}=s\right\}>0$ for some $t$.
} 
$\left\{x_{t}\right\}$ following a Markov process,

$$
x_{t+1}=f\left(x_{t}, \varepsilon_{t}\right),\left\{\varepsilon_{t}\right\} \text { is independently and identically distributed (i.i.d.), }
$$

an endogenous state variable $\left\{y_{t}\right\}$ and a control variable $\left\{c_{t}\right\}$. The optimization problem is

$$
\max _{c_{t}} \sum_{t=0}^{\infty} \beta^{t} U\left(x_{t}, y_{t}, c_{t}\right) \quad \text { such that } y_{t+1}=g\left(y_{t}, c_{t}\right) .
$$

If the optimal policy function is $c_{t}=c\left(x_{t}, y_{t}\right)$, the exogenous state $x_{t}$ and endogenous state $y_{t}$ evolve together as a Markov process:

$$
\left(\begin{array}{l}
x_{t+1} \\
y_{t+1}
\end{array}\right)=\left(\begin{array}{c}
f\left(x_{t}, \varepsilon_{t}\right) \\
g\left(y_{t}, c\left(x_{t}, y_{t}\right)\right)
\end{array}\right) .
$$

Even if the Markov process $x_{t}$ is irreducible, it is typically hard to verify that $\left(x_{t}, y_{t}\right)$ is irreducible. The reason is that in most cases, we can only obtain limited information about $c\left(x_{t}, y_{t}\right)$, instead of having an explicit functional form. This makes it difficult to predict the movement of $\left\{y_{t}\right\}$. The difficulty of verifying the irreducibility condition urges us to find alternative approaches in achieving stability of dynamic systems. This paper proposes the use of stochastic monotonicity as a tool in understanding the structure of dynamic systems. By looking at the stochastic-dominance relationship between state variables $y_{t}$ and $y_{t+1}$, together with the monotonicity of the policy function, we can predict the relationship between $y_{t+1}$ and $y_{t+2}$. Taking this to the limit, we first show the existence of an invariant distribution and then prove the stability of the system. This is motivated by the simple fact that in a deterministic growth model, there is a unique steady-state level $k^{*}$ of capital. If the initial capital $k_{0}$ is less than $k^{*}$, then $k_{0}<k_{1}$, and inductively $k_{t}<k_{t+1}$ for every $t \geq 1$. If the initial capital is greater than $k^{*}$, we obtain a monotonically decreasing sequence of capital. This paper shows that the pattern of monotonic convergence in the deterministic case still holds in the presence of technology shocks.

The idea of using stochastic monotonicity to study dynamics systems is not new. Hopenhayn and Prescott (1992) noted that stochastic monotonicity arises in economic models from the monotonicity of decision rules. They argued that the existence of an invariant distribution can be proved if the state space is compact, which implies compactness of the set of probability measures on the state space. They also provided conditions under which optimal decisions are monotonic functions of the state and induce a monotonic Markov process. 
The main innovation here is the method of constructing a compact subset containing a fixed point in the dynamic system. Since we are dealing with a general state space, the set of all probability distributions on the space may not be compact. This defies the application of most fixed-point theorems. We find that by carefully constructing two boundary distributions, we can actually show that the distributions confined by these two boundary distributions form a compact subset, and this subset is absorbing and contains a fixed point. Based on our method, the idea of stochastic monotonicity can be applied to a wider range of economic dynamic systems. In the last section of the paper, we also discuss some difficulties in applying this method.

The paper is organized as follows. Some useful mathematical results are reviewed in Section 2. The stochastic growth model is introduced in Section 3. Section 4 discusses properties of the transitional operator governing the evolution of output distribution. In particular, continuity and monotonicity of the operator are studied. Section 5 shows the existence, uniqueness and asymptotic stability of the invariant distribution. The final section contains concluding comments.

\section{Definitions and mathematical results}

For any metric space $S$, we denote the Borel $\sigma$-algebra of $S$ by $\mathscr{B}(S)$, and the set of all probability measures on $\mathscr{B}(S)$ by $\Lambda(S)$. If $\lambda$ is a measure on $\mathscr{B}(S)$, then $\|\lambda\|$ is its total variation norm. If $s \in S$, then $\delta_{s}$ is the probability that puts mass 1 at $s$. Let $C_{b}(S)$ be the set of all bounded continuous functions on $S$.

A sequence $\lambda_{n}$ of elements of $\Lambda(S)$ converges weakly (or in distribution) to some $\lambda$ in $\Lambda(S)$ if and only if $\int_{S} f d \lambda_{n}$ converges to $\int_{S} f d \lambda$ for all $f$ in $C_{b}(S)$. Using this notion of convergence, we have a topology defined on $\Lambda(S)$ called the weak topology. In this paper, unless otherwise specified, we use weak topology when we discuss the convergence of distributions.

A transition kernel on $(S, \mathscr{B}(S))$ is a function $Q: S \times \mathscr{B}(S) \mapsto[0,1]$ such that: (1) for each measurable set $A \in \mathscr{B}(S)$, the real valued function $Q(\cdot, A)$ is $\mathscr{B}(S)$ measurable; and (2) for each point $s \in S$, the set function $Q(s, \cdot)$ is a probability measure on $\mathscr{B}(S)$. The number $Q(s, A)$ should be interpreted as the probability that the economic system will move from state $s$ to some state in the set $A$ during one period of time.

A transition kernel defines a linear operator $T$ from bounded measurable functions to 
bounded measurable functions via the formula

$$
(T \phi)(x)=\int \phi(y) Q(x, d y) .
$$

The adjoint $T^{*}: \Lambda(S) \mapsto \Lambda(S)$ of operator $T$ is defined by the formula

$$
\left(T^{*} \lambda\right)(A)=\int Q(x, A) \lambda(d x) .
$$

A transition kernel $Q$ is said to have the Feller property if one of the following equivalent conditions is satisfied.

(1) $T \phi$ is bounded and continuous whenever $\phi$ is.

(2) $T^{*} \lambda_{n}$ converges to $T^{*} \lambda$ whenever $\lambda_{n}$ converges to $\lambda$.

For any two probability measures $\lambda, \tau$ in $\Lambda(\mathbb{R}), \lambda$ (first-order) stochastically dominates $\tau$ if $\int \phi d \lambda \geq \int \phi d \tau$ for all bounded and increasing functions $\phi$. If $\lambda$ dominates $\tau$, we write $\lambda \succeq \tau$ or $\tau \preceq \lambda$. It is known that

$$
\lambda \succeq \tau \Leftrightarrow F_{\lambda}(x) \leq F_{\tau}(x), \quad \text { all } x \in \mathbb{R},
$$

where $F_{\lambda}(\cdot)$ and $F_{\tau}(\cdot)$ are distribution functions of $\lambda$ and $\tau$, respectively. A transition kernel $Q$ on $(\mathbb{R}, \mathscr{B}(\mathbb{R}))$ is called monotonic if it satisfies any of the following equivalent conditions:

(1) $T \phi$ is bounded and increasing if $\phi$ is.

(2) $T^{*} \lambda \succeq T^{*} \tau$, if $\lambda \succeq \tau$.

(3) $Q(x, \cdot) \succeq Q(y, \cdot)$, if $x \geq y$.

Let $M \subseteq \Lambda(S)$ be a subset of probability measures. Then $M$ is tight if for any $\varepsilon>0$, there exists a compact subset $K \subseteq S$ such that $\lambda(K) \geq 1-\varepsilon$ for all $\lambda$ in $M$. If $S$ is complete and separable, then $M$ is tight if and only if the closure of $M$ is weakly compact. 


\section{Stochastic growth model}

This section gives a formulation of the stochastic growth model studied by Brock and Mirman (1972) and Stachurski (2002). At the beginning of period $t$, the representative agent receives income $y_{t}$. In response, a level of consumption $c_{t} \leq y_{t}$ is chosen, yielding current utility $u\left(c_{t}\right)$. The remainder is invested in production, returning output $y_{t+1}=$ $\varepsilon_{t+1} f\left(y_{t}-c_{t}\right)$ in the next period. Here $f$ is the production function and $\varepsilon_{t+1}$ is a nonnegative random variable, representing the production shock at $t+1$. The process then repeats.

Brock and Mirman (1972) assumed that shocks have a bounded distribution to simplify the proof.

Definition 1 A distribution $\mu$ is bounded (both from above and from below) if there are two numbers $a, b, 0<a<b<\infty$, such that support $(\mu) \subseteq[a, b]$.

\subsection{Assumptions}

In this paper, we assume that $u$ and $f$ satisfy standard assumptions, but different from Brock and Mirman, we allow the productivity shock to be unbounded.

Assumption 1 The production function $f: \mathbb{R}_{+} \mapsto \mathbb{R}_{+}$is strictly increasing, strictly concave, differentiable and satisfies the Inada condition

$$
\lim _{x \rightarrow 0} f^{\prime}(x)=+\infty, \lim _{x \rightarrow+\infty} f^{\prime}(x)=0 .
$$

Furthermore, $f(0)=0$.

Assumption 2 The utility function $u: \mathbb{R}_{+} \mapsto \mathbb{R}$ is strictly increasing, strictly concave, differentiable and satisfies $\lim _{c \rightarrow 0} u^{\prime}(c)=+\infty$.

Assumption 3 The productivity shock $\left\{\varepsilon_{t}\right\}_{t=0}^{\infty}$ is a sequence of i.i.d. random variables, with distribution $\mu \in \Lambda\left(\mathbb{R}_{+}\right), 0<E\left(\varepsilon_{t}\right)<\infty$.

\subsection{Transition kernel}

The conditional distribution of next-period output $y^{\prime}$ given current output $y$ and consumption $c$ is

$$
\operatorname{Pr}\left(y^{\prime} \in B\right)=\mu\left(\frac{B}{f(y-c)}\right), \quad \text { if } y-c>0, \text { for all } B \text { in } \mathscr{B}\left(\mathbb{R}_{+}\right)
$$


where $\frac{B}{f(y-c)}=\left\{\frac{y^{\prime}}{f(y-c)}: y^{\prime} \in B\right\}$. However, if $y-c=0$, since $f(0)=0$, we know that $\operatorname{Pr}\left(y^{\prime}=0\right)=1$ and 0 is an absorbing state.

Let $Q(y, c ; B)$ denote the probability that the next-period output is in $B$, given that the current income is $y$ and consumption is $c$.

$$
Q(y, c ; B)= \begin{cases}\mu\left(\frac{B}{f(y-c)}\right), & y-c>0 \\ 1, & y-c=0,0 \in B \\ 0, & y-c=0,0 \notin B\end{cases}
$$

\subsection{The optimal policy}

Future utility is discounted at rate $\beta \in(0,1)$. The agent selects a sequence $\left\{c_{t}\right\}_{t=0}^{\infty}$ to maximize expected utility.

$$
\max E\left(\sum_{t=0}^{\infty} \beta^{t} u\left(c_{t}\right)\right) \quad \text { such that } y_{t}=\varepsilon_{t} f\left(y_{t-1}-c_{t-1}\right), 0 \leq c_{t} \leq y_{t} .
$$

Using dynamic programming, the maximization problem can be solved recursively. Let the value function be $V(y)$ and the policy function of consumption be $c_{t}=g\left(y_{t}\right)$. The following results are well known.

THEOREM 1 Let $u, f, \mu$ satisfy assumptions $1-3$; then the following results hold.

(1) The value function $V$ is finite and satisfies the Bellman equation

$$
V(y)=\max _{0 \leq c \leq y}\left\{u(c)+\beta \int_{0}^{\infty} V\left(y^{\prime}\right) Q\left(y, c ; d y^{\prime}\right)\right\} .
$$

(2) There exists a unique optimal policy $g$, such that

$$
V(y)=u(g(y))+\beta \int_{0}^{\infty} V(y) Q\left(y, g(y) ; d y^{\prime}\right)
$$

(3) The value function is non-decreasing, concave and differentiable, with

$$
V^{\prime}(y)=u^{\prime}(g(y))
$$

(4) The optimal policy $g$ is continuous, $0<g(y)<y$, for all $y>0$, and both $y \mapsto g(y)$ and $y \mapsto y-g(y)$ are strictly increasing (savings and consumption both increase with income). 
Proof. For parts $(1-3)$, see Mirman and Zilcha (1975), pp. 331-332. Part (4) is proved in Stokey et al. (1989), Exercises 10.1, pp. 288-289.

\section{Properties of the transition kernel}

We utilize an operator-theoretical approach to the invariant distribution problem. Substituting the optimal policy into the production relation yields a closed-loop law of motion

$$
y_{t+1}=\varepsilon_{t+1} f\left(y_{t}-g\left(y_{t}\right)\right)
$$

To simplify notation in later discussion, we define

$$
h(y) \equiv f(y-g(y)) .
$$

Note that function $h$ is strictly increasing, continuous, and $h(0)=0$. If there is no confusion, we also use $Q(y, B)$ to denote $Q(y, g(y) ; B)$. Since $h$ is continuous, it is intuitive that the transitional kernel $Q(\cdot, \cdot)$ satisfies the Feller property. Interested readers can find a proof in Theorem 8.9 and Exercise 8.10 (pp. 234-237) in Stokey et al. (1989).

Now we study another important property of $T^{*}$, namely, monotonicity.

Lemma 1 The transition kernel $Q$ in the stochastic growth model is monotonic.

Proof. We need to show that $x, y \in \mathbb{R}_{+}$and $x \geq y$ implies $Q(x, \cdot) \succeq Q(y, \cdot)$. For any $z \geq 0$

a) if $x>y=0$, then

$$
Q(x,[0, z])=\mu([0, z / h(x)]) \leq 1=Q(0,[0, z]) .
$$

b) if $x \geq y>0$, then, since $h$ is an increasing function,

$$
Q(x,[0, z])=\mu([0, z / h(x)]) \leq \mu([0, z / h(y)])=Q(y,[0, z]) .
$$

Therefore, $Q$ is monotonic.

For a stochastic growth model, the state of the economic system can be represented by a probability distribution of output $y_{t}$. Suppose the distribution of $y_{t}$ is $\lambda_{t}$, then the distribution of $y_{t+1}$ is $T^{*} \lambda_{t}$. Recall that $T^{*}$ is an operator $\Lambda\left(\mathbb{R}_{+}\right) \mapsto \Lambda\left(\mathbb{R}_{+}\right)$. In the language of dynamic systems, the time path of the system is $\left\{\lambda_{t}\right\}_{t=0}^{\infty}$, with law of motion $\lambda_{t+1}=T^{*} \lambda_{t}$. Starting from any initial distribution $\lambda_{0}$, we can obtain the trajectory of $\lambda_{0}$ by operator $T^{*}$. 


\section{Invariant probability distribution}

For a dynamic stochastic system, we usually look at an invariant(or, stationary) distribution to study the long-run behavior of the process. The distribution of a state variable is invariant, even though the state variable itself is stochastic over time.

Definition $2 \lambda \in \Lambda\left(\mathbb{R}_{+}\right)$is called an invariant probability distribution if it is a fixed point of the operator $T^{*}$, that is $\lambda=T^{*} \lambda$.

LEMma $2 \delta_{0}$ is an invariant probability distribution.

Proof. Since $Q(0,\{0\})=1, T^{*} \delta_{0}=\delta_{0}$.

Lemma 3 If $\mu(0)>0$, then $\delta_{0}$ is the unique invariant probability distribution, and for any initial distribution $\lambda_{0}$,

$$
\lim _{t \rightarrow \infty} \lambda_{t}(\{0\})=1
$$

Proof. Since $\lambda_{t}((0, \infty))=\lambda_{0}((0, \infty))(1-\mu(\{0\}))^{t}$, the second statement is easily proven. We can check $\lim _{t \rightarrow \infty}\left\|\lambda_{t}-\delta_{0}\right\|=0$, which implies $\delta_{0}$ is the unique fixed point.

Obviously, if $\mu(\{0\})>0$, the economy will die out with probability one. This is an uninteresting case. From now on, we impose an assumption on $\mu$.

Assumption $4 \quad \mu(\{0\})=0$.

When $\mu(\{0\})=0$, we can restrict the operator $T^{*}$ on $\Lambda\left(\mathbb{R}_{++}\right)$. This is because part (4) of Theorem 1 implies that $T^{*}\left(\Lambda\left(\mathbb{R}_{++}\right)\right)$is contained in $\Lambda\left(\mathbb{R}_{++}\right)$.

\subsection{Existence of a fixed point in $\Lambda\left(\mathbb{R}_{++}\right)$}

In order to obtain a fixed point different from zero, we need another condition on $\mu$.

Assumption $5 \quad \int x^{-1} \mu(d x)<\infty$

LEMma 4 There exists a number $s>0$, such that $E(s / \varepsilon)=1, \mu((0, s])>0$, and $\mu([s, \infty))>$ 0 . 
Proof. Take $s=\left[E\left(\varepsilon^{-1}\right)\right]^{-1}$. We must show $\mu((0, s])>0$ and $\mu([s, \infty))>0$. By contradiction, suppose that $\mu((0, s])=0$. Then $\varepsilon>s($ a.s. $)$ implies $E\left(\varepsilon^{-1}\right)>s^{-1}$. This contradicts the definition of $s$. The proof for $\mu([s, \infty))>0$ is similar.

If we redefine $\varepsilon^{*}=\varepsilon / s, f^{*}(\cdot)=s f(\cdot)$, and $h^{*}(\cdot)=s h(\cdot)$, then the optimal growth problem does not change in an essential way. So without loss of generality, we may assume that $\left[E\left(\varepsilon^{-1}\right)\right]^{-1}=1$.

LEMMA 5 There exists a number $\underline{y}>0$, such that $h(y)>y$ for all $y \in(0, \underline{y}$.

Proof. The first-order condition in the optimization problem is

$$
u^{\prime}(g(y))=\beta f^{\prime}\left(f^{-1}(h(y))\right) \int_{0}^{\infty} V^{\prime}(h(y) \varepsilon) \varepsilon \mu(d \varepsilon) .
$$

The first-order condition (FOC) and the envelope condition $V^{\prime}(y)=u^{\prime}(g(y))$ imply

$$
V^{\prime}(y)=\beta f^{\prime}\left(f^{-1}(h(y))\right) \int_{0}^{\infty} V^{\prime}(h(y) \varepsilon) \varepsilon \mu(d \varepsilon) .
$$

Then we have

$$
\begin{aligned}
\frac{V^{\prime}(y)}{V^{\prime}(h(y))} & =\frac{\beta f^{\prime}\left(f^{-1}(h(y))\right) \int_{0}^{\infty} V^{\prime}(h(y) \varepsilon) \varepsilon \mu(d \varepsilon)}{V^{\prime}(h(y))} \\
& \geq \frac{\beta f^{\prime}\left(f^{-1}(h(y))\right) \int_{(0,1]} V^{\prime}(h(y) \varepsilon) \varepsilon \mu(d \varepsilon)}{V^{\prime}(h(y))} \\
& \geq \beta f^{\prime}\left(f^{-1}(h(y))\right) \int_{(0,1]} \varepsilon \mu(d \varepsilon) .
\end{aligned}
$$

Since $\lim _{y \rightarrow 0} f^{\prime}\left(f^{-1}(h(y))\right)=\infty$ and $\int_{(0,1]} \varepsilon \mu(d \varepsilon)>0$, there exits a $\underline{y}>0$, such that if $y \leq \underline{y}$, $\frac{V^{\prime}(y)}{V^{\prime}(h(y))}>1 \Rightarrow h(y)>y$.

Lemma 6 If $z \in(0, \underline{y}]$ and $\tau_{z}$ is the uniform distribution on the interval $(0, z]$, that is, for $0 \leq a \leq b \leq z, \tau_{z}([a, b])=(b-a) / z$. We have

$$
T^{*} \tau_{z} \succeq \tau_{z} .
$$

Proof. It is sufficient to show that for any $m \in(0, z],\left(T^{*} \tau_{z}\right)((0, m]) \leq \tau_{z}((0, m])$. Applying the definition of $T^{*}$, and using the fact that $h(y)>y$, we find that

$$
\begin{aligned}
\left(T^{*} \tau_{z}\right)((0, m]) & =\int_{0}^{z} \mu((0, m / h(y)]) \tau_{z}(d y) \\
& \leq \int_{0}^{z} \mu\left(\left(0, m y^{-1}\right]\right) \tau_{z}(d y) .
\end{aligned}
$$




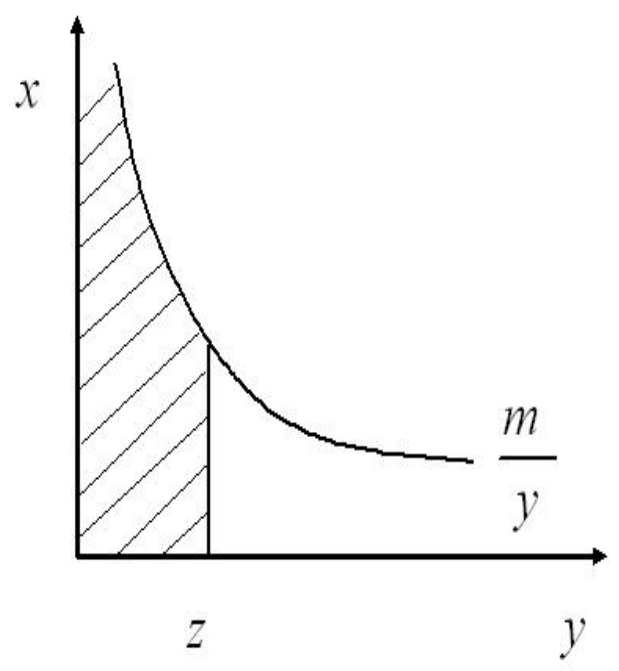

Figure 1:

From Figure 1, applying Fubini's theorem and using the fact that $E\left(\varepsilon^{-1}\right)=1$ after normalization, we obtain

$$
\begin{aligned}
\int_{0}^{z} \mu\left(\left(0, m y^{-1}\right]\right) \tau_{z}(d y) & =\int_{0}^{z} \int_{0}^{m y^{-1}} \mu(d x) \tau_{z}(d y) \\
& \leq \int_{0}^{\infty} \int_{0}^{m x^{-1}} \tau_{z}(d y) \mu(d x) \\
& \leq \int_{0}^{\infty} m x^{-1} z^{-1} \mu(d x) \\
& =m z^{-1} \\
& =\tau_{z}((0, m])
\end{aligned}
$$

Therefore, $T^{*} \tau_{z} \succeq \tau_{z}$.

For each $z \in(0, \underline{y}]$, we define subset $B_{z}$ of $\Lambda\left(\mathbb{R}_{++}\right)$by

$$
B_{z}=\left\{\tau \in \Lambda\left(\mathbb{R}_{++}\right): \tau \succeq \tau_{z}\right\}
$$

Lemma $7 T^{*}\left(B_{z}\right) \subseteq B_{z}$ and $B_{z}$ is a closed subset of $\Lambda\left(\mathbb{R}_{++}\right)$. 
Proof. First part: Choose any $\tau \in B_{z}$, then $\tau \succeq \tau_{z}$. By the monotonicity of $T^{*}$, $T^{*} \tau \succeq T^{*} \tau_{z} \succeq \tau_{z}$, and therefore $T^{*} \tau \in B_{z}$.

Second part: Choose a sequence $\left\{\tau_{n}\right\}_{n=1}^{\infty}$ in $B_{z}$ converging to $\tau$. We show that $\tau \in B_{z}$. Let $F_{\tau}, F_{\tau_{n}}$, and $F_{\tau_{z}}$ be the distribution functions of $\tau, \tau_{n}$, and $\tau_{z}$, respectively. If $y$ is a continuity point of $F_{\tau}$, then $F_{\tau}(y)=\lim _{n \rightarrow \infty} F_{\tau_{n}}(y) \leq F_{\tau_{z}}(y)$. Since continuity points are dense in $\mathbb{R}, F_{\tau}(y) \leq F_{\tau_{z}}(y)$ for all $y$.

So far, we have found the lower boundary $\left(\tau_{z}\right)$ of the dynamic system. Now we move on to find the upper boundary. Recall that $h(y)=f(y-g(y)) \leq f(y)$ and we assume that $\lim _{y \rightarrow \infty} f^{\prime}(y)=0$. Therefore, $\lim _{y \rightarrow \infty} h(y) / y=0$. There exists a $\bar{y}>0$, such that

$$
\forall y \geq \bar{y}, \quad h(y)<y(E(\varepsilon))^{-1} .
$$

For any $z \in[\bar{y}, \infty)$, let $\lambda^{z}$ be the probability distribution with density function ${ }^{2}$

$$
\text { density at } y= \begin{cases}z y^{-2}, & y \geq z \\ 0, & \text { otherwise }\end{cases}
$$

LEMMA $8 \lambda^{z} \succeq T^{*} \lambda^{z}$.

Proof. The idea of the proof is similar to Lemma 6. Choose $m \in[z, \infty)$. Applying the definition of $T^{*}$ and using the fact that $h(y)<y(E(\varepsilon))^{-1}$, we find that

$$
\begin{aligned}
T^{*} \lambda^{z}([m, \infty)) & =\int_{z}^{\infty} \mu([m / h(y), \infty)) \lambda^{z}(d y) \\
& \leq \int_{z}^{\infty} \mu\left(\left[m E(\varepsilon) y^{-1}, \infty\right)\right) \lambda^{z}(d y) \\
& =\int_{z}^{\infty} \int_{m E(\varepsilon) y^{-1}}^{\infty} \mu(d x) \lambda^{z}(d y) .
\end{aligned}
$$

Applying the definition of $\lambda^{z}$ and using Fubini's theorem again, we obtain

$$
\begin{aligned}
\int_{z}^{\infty} \int_{m E(\varepsilon) y^{-1}}^{\infty} \mu(d x) \lambda^{z}(d y) & \leq \int_{0}^{\infty} \int_{m E(\varepsilon) x^{-1}}^{\infty} z y^{-2} d y \mu(d x) \\
& =\int_{0}^{\infty} x z(m E(\varepsilon))^{-1} \mu(d x) \\
& =z m^{-1}=\lambda^{z}([m, \infty)) .
\end{aligned}
$$

\footnotetext{
${ }^{2}$ It is easy to verify that $\int_{z}^{\infty} z y^{-2} d y=1$.
} 


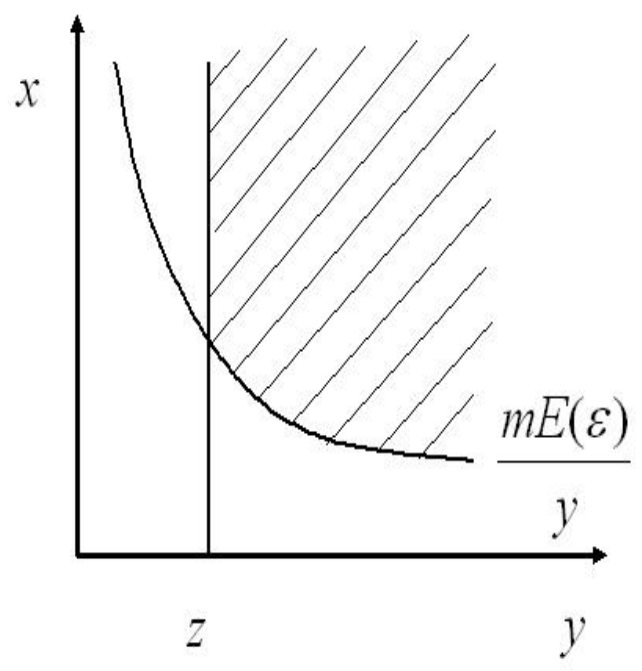

Figure 2:

Therefore, $T^{*} \lambda^{z}([m, \infty)) \leq \lambda^{z}([m, \infty))$, and hence $T^{*} \lambda^{z} \preceq \lambda^{z}$.

Similar to the definition of $B_{z}$, for any $z \in[\bar{y}, \infty)$, we define

$$
B^{z}=\left\{\lambda \in \Lambda\left(\mathbb{R}_{++}\right): \lambda \preceq \lambda^{z}\right\} .
$$

Similar to Lemma $7, T^{*}\left(B^{z}\right) \subseteq B^{z} . B^{z}$ is used as an upper boundary in finding an invariant distribution.

Lemma 9 Choose $z_{1} \in(0, \underline{y}], z_{2} \in[\bar{y}, \infty), z_{1}<z_{2}$, then $B_{z_{1}} \cap B^{z_{2}}$ is a non-empty, convex and compact subset of $\Lambda\left(\mathbb{R}_{++}\right)$.

Proof. Convexity, closedness and non-emptiness are obvious. To show compactness in the weak topology, we only need the condition that $B_{z_{1}} \cap B^{z_{2}}$ is tight.

For any $\delta>0$, if $\lambda \in B_{z_{1}} \cap B^{z_{2}}$, then

$$
\begin{aligned}
\lambda\left(\left(0, \frac{1}{2} \delta z_{1}\right)\right) & \leq \tau_{z_{1}}\left(\left(0, \frac{1}{2} \delta z_{1}\right)\right)=\frac{1}{2} \delta \\
\lambda\left(\left(2 z_{2} \delta^{-1}, \infty\right)\right) & \leq \lambda^{z_{2}}\left(\left(2 z_{2} \delta^{-1}, \infty\right)\right)=\frac{1}{2} \delta
\end{aligned}
$$

Therefore, $\lambda\left(\left[\frac{1}{2} \delta z_{1}, 2 z_{2} \delta^{-1}\right]\right) \geq 1-\frac{1}{2} \delta-\frac{1}{2} \delta=1-\delta$. Thus, $B_{z_{1}} \cap B^{z_{2}}$ is tight. 
Proposition 1 Under Assumptions 1-5, there exists an invariant probability measure in $\Lambda\left(\mathbb{R}_{++}\right)$.

Proof. First, we show that $T^{*}\left(B_{z_{1}} \cap B^{z_{2}}\right) \subseteq B_{z_{1}} \cap B^{z_{2}}$.

$$
\begin{aligned}
T^{*}\left(B_{z_{1}} \cap B^{z_{2}}\right) & \subseteq T^{*}\left(B_{z_{1}}\right) \cap T^{*}\left(B^{z_{2}}\right) \\
& \subseteq B_{z_{1}} \cap B^{z_{2}}
\end{aligned}
$$

Since $Q(\cdot, \cdot)$ satisfies the Feller property, $T^{*}$ is a continuous operator from $\Lambda\left(\mathbb{R}_{++}\right)$to $\Lambda\left(\mathbb{R}_{++}\right)$under weak topology. By the Brouwer-Schauder-Tychonoff theorem (see p. 550, Aliprantis and Border (1999)), $T^{*}$ has a fixed point in $B_{z_{1}} \cap B^{z_{2}}$.

\subsection{Stability of $T^{*}$}

As stated previously, $\left\{\Lambda\left(\mathbb{R}_{++}\right), T^{*}\right\}$ constitutes a dynamic system. We now study the stability of the system.

DeFinition 3 The above system is globally asymptotically stable if there is a fixed point $\lambda^{*} \in \Lambda\left(\mathbb{R}_{++}\right)$and, for any other initial distribution $\lambda_{0}, \lim _{t \rightarrow \infty}\left(T^{*}\right)^{t} \lambda_{0}=\lambda^{*}$.

Lemma 10 Choose $z_{1} \in(0, \underline{y}], z_{2} \in[\bar{y}, \infty), z_{1}<z_{2} .\left\{\left(T^{*}\right)^{t} \tau_{z_{1}}\right\}_{t=1}^{\infty}$ and $\left\{\left(T^{*}\right)^{t} \lambda^{z_{2}}\right\}_{t=1}^{\infty}$ converge to (possibly different) fixed points.

Proof. From previous arguments, $\left\{\left(T^{*}\right)^{t} \tau_{z_{1}}\right\}$ is monotonically increasing and belongs to the compact set $B_{z_{1}} \cap B^{z_{2}}$. It follows that there is a convergent subsequence $\left\{\left(T^{*}\right)^{t_{i}} \tau_{z_{1}}\right\}_{i=1}^{\infty}$ of $\left\{\left(T^{*}\right)^{t} \tau_{z_{1}}\right\}_{t=1}^{\infty}$. We denote the limit of this subsequence by $\lambda^{*}$.

We need to show that the sequence itself converges to $\lambda^{*}$ and that $\lambda^{*}$ is a fixed point. If $y$ is a continuity point of distribution function $F_{\lambda^{*}}$, then

$$
\lim _{i \rightarrow \infty} F_{\left(\left(T^{*}\right)^{t_{i}} \tau_{z_{1}}\right)}(y)=F_{\lambda^{*}}(y) .
$$

Since $\left\{F_{\left(\left(T^{*}\right)^{t} \tau_{z_{1}}\right)}(y)\right\}_{t=1}^{\infty}$ is a monotonic sequence, we obtain $\lim _{t \rightarrow \infty} F_{\left(\left(T^{*}\right)^{t} \tau_{z_{1}}\right)}(y)=F_{\lambda^{*}}(y)$, which implies that $\lim _{t \rightarrow \infty}\left(T^{*}\right)^{t} \tau_{z_{1}}=\lambda^{*}$.

To show that $\lambda^{*}$ is a fixed point, taking the limit in $\left(T^{*}\right)^{t+1} \tau_{z_{1}}=\left(T^{*}\right)\left(T^{* t} \tau_{z_{1}}\right)$ yields $\lambda^{*}=\left(T^{*}\right)\left(\lambda^{*}\right)$.

The proof for $\left(T^{*}\right)^{t} \lambda^{z_{2}}$ is similar. 
Proposition 2 Under Assumptions 1-5, $\left\{\Lambda\left(\mathbb{R}_{++}\right), T^{*}\right\}$ is globally asymptotically stable if and only if $T^{*}$ has a unique fixed point.

Proof. If $\left\{\Lambda\left(\mathbb{R}_{++}\right), T^{*}\right\}$ is globally asymptotically stable, it is obvious that the fixed point of $T^{*}$ is unique.

Conversely, suppose that $T^{*}$ has a unique fixed point, denoted by $\lambda^{*}$. From Lemma 10 , for arbitrary $z_{1} \in(0, \underline{y}], z_{2} \in[\bar{y}, \infty)$,

$$
\begin{aligned}
\left(T^{*}\right)^{t} \tau_{z_{1}} & \rightarrow \lambda^{*} \\
\left(T^{*}\right)^{t} \lambda^{z_{2}} & \rightarrow \lambda^{*}
\end{aligned}
$$

This implies that $\left(T^{*}\right)^{t} \lambda_{0} \rightarrow \lambda^{*}$, for every $\lambda_{0} \in B_{z_{1}} \cap B^{z_{2}}$. Now choose any $\lambda \in \Lambda\left(\mathbb{R}_{++}\right)$ and define $\lambda_{z_{1}}^{z_{2}} \in \Lambda\left(\mathbb{R}_{++}\right)$by

$$
\begin{aligned}
\lambda_{z_{1}}^{z_{2}}\left(\left(0, z_{1}\right)\right) & =0, \quad \lambda_{z_{1}}^{z_{2}}\left(\left\{z_{1}\right\}\right)=\left(\left(0, z_{1}\right]\right) \\
\lambda_{z_{1}}^{z_{2}}\left(\left(z_{2}, \infty\right)\right) & =0, \quad \lambda_{z_{1}}^{z_{2}}\left(\left\{z_{2}\right\}\right)=\lambda\left(\left[z_{2}, \infty\right)\right) \\
\lambda_{z_{1}}^{z_{2}}((a, b)) & =\lambda((a, b)), \quad \text { if } z_{1}<a<b<z_{2}
\end{aligned}
$$

Then $\lambda_{z_{1}}^{z_{2}} \in B_{z_{1}} \cap B^{z_{2}}$, and by choosing $z_{1}$ small enough and $z_{2}$ large enough, $\left\|\lambda_{z_{1}}^{z_{2}}-\lambda\right\|$ can be made arbitrarily small.

To show $\left(T^{*}\right)^{t} \lambda \rightarrow \lambda^{*}$, equivalently, we should show that for any $\phi \in C_{b}\left(\mathbb{R}_{++}\right)$

$$
\lim _{t \rightarrow \infty} \int \phi(y)\left(\left(T^{*}\right)^{t} \lambda\right)(d y)=\int \phi(y) \lambda^{*}(d y) .
$$

For any small $\delta>0$, choose $z_{1} \in(0, \underline{y}], z_{2} \in[\bar{y}, \infty)$ such that $\left\|\lambda_{z_{1}}^{z_{2}}-\lambda\right\| \leq \delta(2\|\phi\|)^{-1}$.

Then choose $N$, such that $t \geq N$ implies

$$
\left|\int \phi(y)\left(\left(T^{*}\right)^{t} \lambda_{z_{1}}^{z_{2}}\right)(d y)-\int \phi(y) \lambda^{*}(d y)\right| \leq \frac{1}{2} \delta .
$$

If $t \geq N$, we have

$$
\begin{aligned}
& \left|\int \phi(y)\left(\left(T^{*}\right)^{t} \lambda\right)(d y)-\int \phi(y) \lambda^{*}(d y)\right| \\
\leq & \left|\int \phi(y)\left(\left(T^{*}\right)^{t} \lambda\right)(d y)-\int \phi(y)\left(\left(T^{*}\right)^{t} \lambda_{z_{1}}^{z_{2}}\right)(d y)\right| \\
& +\left|\int \phi(y)\left(\left(T^{*}\right)^{t} \lambda_{z_{1}}^{z_{2}}\right)(d y)-\int \phi(y) \lambda^{*}(d y)\right|
\end{aligned}
$$




$$
\begin{aligned}
& \leq\left|\int\left(T^{t} \phi\right)(y) \lambda(d y)-\int\left(T^{t} \phi\right)(y) \lambda_{z_{1}}^{z_{2}}(d y)\right|+\frac{1}{2} \delta \\
& \leq\left\|T^{t} \phi\right\| \cdot\left\|\lambda_{z_{1}}^{z_{2}}-\lambda\right\|+\frac{1}{2} \delta \\
& \leq\|\phi\| \cdot\left\|\lambda_{z_{1}}^{z_{2}}-\lambda\right\|+\frac{1}{2} \delta \leq \delta .
\end{aligned}
$$

The equivalence result is thus established.

\subsection{Uniqueness of the fixed point in $\Lambda\left(\mathbb{R}_{++}\right)$}

The above proposition implies that in order to achieve global asymptotic stability, we must prove the uniqueness of the fixed point of $T^{*}$.

Our proof strategy is to utilize a process called the 'reverse Markov process', first introduced by Brock and Mirman (1972). The 'reverse Markov process' is described by the following transition function (note that the time index is backwards)

$$
y_{t-1}=h^{-1}\left(y_{t} / \varepsilon_{t}\right), \quad y_{t} \in(0, \infty), \quad t \leq 0,
$$

where $\left\{\varepsilon_{t}\right\}_{t=0}^{-\infty}$ is a sequence of i.i.d. shocks. We show that, starting at any initial condition, this process will almost surely converge to 0 or $\infty$. This feature provides us with a contradiction when we assume the existence of more than one invariant distribution.

Recall that $y_{t+1}=h\left(y_{t}\right) \varepsilon_{t+1}$; therefore, $y_{t}=h^{-1}\left(y_{t+1} / \varepsilon_{t+1}\right)$. Also recall that there is $\underline{y}>0$, such that

$$
y \in(0, \underline{y}] \Rightarrow h(y)>y .
$$

For any $z \in(0, \underline{y}]$, we consider first the set $(0, z]$. The following rule

$$
y_{t-1}= \begin{cases}h^{-1}\left(\min \left(y_{t} / \varepsilon_{t}, h(z)\right)\right), & y_{t}<z \\ z, & y_{t}=z\end{cases}
$$

specifies a transition kernel on $(0, z]$. Thus, we can define a Markov process $\left\{y_{t}\right\}_{t=0}^{-\infty}$ using Eq. (13), an i.i.d. sequence of shocks $\left\{\varepsilon_{t}\right\}_{t=0}^{-\infty}$, and any initial random variable $y_{0}$ with range in $(0, z]$. Note that this Markov process is a modification of the 'reverse Markov process', in which once $y_{t}$ is larger than or equal to $z$, it is redefined to be $z$ and stays there afterwards.

Lemma 11 The above Markov process on $(0, z]$ is a super-martingale, that is

$$
E\left[y_{t-1} \mid y_{t}, y_{t+1}, y_{t+2} \ldots y_{0}\right] \leq y_{t} .
$$


Proof. Since if $y_{t}=z$, then $E\left(y_{t-1} \mid y_{t}\right)=z=y_{t}$, it suffices to show that

$$
E\left[h^{-1}(\min (y / \varepsilon, h(z))) \mid y\right] \leq y, \quad \text { for any } y<z .
$$

Using the fact that $h^{-1}(y)<y$, for $y \leq h(z)$, we find

$$
\begin{aligned}
E\left[h^{-1}(\min (y / \varepsilon, h(z))) \mid y\right] & <E_{\varepsilon}[\min (y / \varepsilon, h(z)) \mid y] \\
& \leq E_{\varepsilon}[y / \varepsilon \mid y] \\
& =y .
\end{aligned}
$$

LEMma 12 For any $z \leq \underline{y}$ and any initial random variable $y_{0}$ taking values in $(0, z)$ with probability 1 ,

$$
\operatorname{Pr}\left(y_{t}<z, \forall t \leq 0\right) \geq \operatorname{Pr}\left(\lim _{t \rightarrow(-\infty)} y_{t}<z\right) \geq \mu([1,+\infty))
$$

Proof. First note that $\left\{y_{t}\right\}_{t=0}^{-\infty}$ is a non-negative super-martingale taking values in $(0, z]$. By the martingale convergence theorem, $\operatorname{Pr}\left(\lim _{t \rightarrow \infty} y_{t}\right.$ exists $)=1$. We set an initial random variable $x$ to be

$$
x=h^{-1}(\min (z / \varepsilon, h(z))), \quad \varepsilon \text { has distribution } \mu .
$$

We show that for any $y_{0}, \operatorname{Pr}\left(\lim _{t \rightarrow(-\infty)} y_{t}<z\right) \geq \mu([1,+\infty))$. Let $y=\lim _{t \rightarrow(-\infty)} y_{t}$ and $\lambda_{y_{t}}$, $\lambda_{x}$ be the distributions of $y_{t}$ and $x$, respectively.

$$
\begin{aligned}
\lambda_{y_{0}}((0, z))=1 & \Rightarrow \lambda_{y_{0}} \text { is dominated by } \delta_{z} \\
& \Rightarrow \lambda_{y_{-1}} \text { is dominated by } \lambda_{x} \\
& \Rightarrow \lambda_{y_{t}} \text { is dominated by } \lambda_{x}, t \leq-1 \\
& \Rightarrow \lambda_{y} \text { is dominated by } \lambda_{x} \\
& \Rightarrow \operatorname{Pr}(y<z) \geq \operatorname{Pr}(x<z) \geq \mu([1,+\infty)) \\
& \Rightarrow \operatorname{Pr}\left(\lim _{t \rightarrow(-\infty)} y_{t}<z\right) \geq \mu([1,+\infty)) .
\end{aligned}
$$

Because $z$ is absorbing, $\operatorname{Pr}\left(y_{t}<z, \forall t \leq 0\right) \geq \operatorname{Pr}\left(\lim _{t \rightarrow(-\infty)} y_{t}<z\right)$. 
Also recall that $y>E(\varepsilon) h(y)$, for $y \geq \bar{y}\left(\right.$ or $h^{-1}(y)>E(\varepsilon) y$, for $\left.y \geq h(\bar{y})\right)$. Now we consider the set $[z, \infty)$, where $z \geq \bar{y}$. Using an i.i.d. sequence of shocks $\left\{\varepsilon_{t}\right\}_{t=0}^{-\infty}$, the following transition function

$$
y_{t-1}= \begin{cases}h^{-1}\left(\max \left(y_{t} / \varepsilon_{t}, h(z)\right)\right), & y_{t}>z \\ z, & y_{t}=z\end{cases}
$$

specifies a Markov process on $[z, \infty)$. This corresponds to a Markov process on $(0,1 / z]$,

$$
x_{t-1}= \begin{cases}\frac{1}{h^{-1}\left(\max \left(\left(x_{t} \varepsilon_{t}\right)^{-1}, h(z)\right)\right)}, & x_{t}<1 / z \\ 1 / z, & x_{t}=1 / z\end{cases}
$$

Lemma 13 The Markov process $\left\{x_{t}\right\}_{t=0}^{-\infty}$ defined above is a super-martingale.

Proof. As in the proof of Lemma 11, it suffices to show that $E\left[\frac{1}{h^{-1}\left(\max \left((x \varepsilon)^{-1}, h(z)\right)\right)} \mid x\right] \leq$ $x$, for $x<1 / z$. Using the fact that $h^{-1}(y)>E(\varepsilon) y$, for $y \geq h(\bar{y})$, we obtain

$$
\begin{aligned}
E\left[\frac{1}{h^{-1}\left(\max \left((x \varepsilon)^{-1}, h(z)\right)\right)} \mid x\right] & \leq E_{\varepsilon}\left[\frac{1}{E(\varepsilon) \max \left((x \varepsilon)^{-1}, h(z)\right)} \mid x\right] \\
& \leq E_{\varepsilon}\left[\frac{1}{E(\varepsilon)(x \varepsilon)^{-1}} \mid x\right] \\
& =x .
\end{aligned}
$$

Using the same proof as in LEMma 12, we also know that for any initial random variable $x_{0}$ taking values in $(0,1 / z)$ with probability 1 ,

$$
\operatorname{Pr}\left(x_{t}<1 / z, \forall t \leq 0\right) \geq \mu((0,1])
$$

Returning to the transition kernel in Eq. (16), it is evident that for any initial random variable $y_{0}$ taking values in $(z, \infty)$, and the corresponding Markov process $\left\{y_{t}\right\}_{t=0}^{-\infty}$,

$$
\operatorname{Pr}\left(y_{t}>z, \forall t \leq 0\right) \geq \mu((0,1]) .
$$

Now we study the 'reverse Markov process'. Let us assume that $\mu$ is either unbounded from below or unbounded from above. For bounded shocks, we can refer to the uniqueness proof of Brock and Mirman (1972). 
Lemma 14 Suppose the shock $\varepsilon$ is unbounded. Choose any number $z_{1} \in(0, y]$ and any number $z_{2} \in[\bar{y}, \infty)$, then there is a $\pi>0$, such that for all $y_{0}, \operatorname{Pr}\left(y_{t} \in\left(0, z_{1}\right) \cup\left(z_{2}, \infty\right), \forall t \leq\right.$ $-1)>\pi$, where $\left\{y_{t}\right\}_{t=0}^{-\infty}$ is the 'reverse Markov process'.

Proof. If $\mu$ is unbounded from above, that is $\mu((N, \infty))>0$, for all $N>0$, set

$$
\pi=\min \left\{\mu([1,+\infty)), \mu((0,1]), \mu\left(\left(z_{2} / h\left(z_{1}\right), \infty\right)\right)\right\} \cdot \min \{\mu([1,+\infty)), \mu((0,1])\}>0
$$

Then we prove that $\operatorname{Pr}\left(y_{t} \in\left(0, z_{1}\right) \cup\left(z_{2}, \infty\right), \forall t \leq-1\right)>\pi$.

(1) If $y_{0} \in\left(0, z_{1}\right)$, then

$$
\begin{aligned}
\operatorname{Pr}\left(y_{t} \in\left(0, z_{1}\right) \cup\left(z_{2}, \infty\right), \forall t \leq-1\right) & \geq \operatorname{Pr}\left(y_{t} \in\left(0, z_{1}\right), \forall t \leq-1\right) \\
& \geq \mu([1,+\infty)) \\
& \geq \pi
\end{aligned}
$$

(2) If $y_{0} \in\left(z_{1}, z_{2}\right]$, then

$$
\begin{aligned}
\operatorname{Pr}\left(y_{t} \in\left(0, z_{1}\right) \cup\left(z_{2}, \infty\right), \forall t \leq-1\right) & \geq \operatorname{Pr}\left(y_{t} \in\left(0, z_{1}\right), \forall t \leq-1\right) \\
& \geq \operatorname{Pr}\left(y_{-1} \in\left(0, z_{1}\right)\right) \cdot \mu([1, \infty)) \\
& \geq \mu\left(\left(z_{2} / h\left(z_{1}\right), \infty\right)\right) \cdot \mu([1, \infty)) \\
& \geq \pi
\end{aligned}
$$

(3) If $y_{0} \in\left(z_{2}, \infty\right)$, then

$$
\begin{aligned}
\operatorname{Pr}\left(y_{t} \in\left(0, z_{1}\right) \cup\left(z_{2}, \infty\right), \forall t \leq-1\right) & \geq \operatorname{Pr}\left(y_{t} \in\left(z_{2}, \infty\right), \forall t \leq-1\right) \\
& \geq \mu((0,1]) \\
& \geq \pi
\end{aligned}
$$

The case for which $\varepsilon$ is unbounded from below is similar.

Now we come to the central lemma of this section.

Lemma 15 For any $z_{1} \in(0, \underline{y}]$ and $z_{2} \in[\bar{y}, \infty)$, if $\left\{y_{t}\right\}_{t=0}^{-\infty}$ is the 'reverse Markov process', then

$$
\operatorname{Pr}\left(\exists \bar{t}<0, \quad \text { such that } y_{t} \in\left(0, z_{1}\right) \cup\left(z_{2}, \infty\right), \forall t \leq \bar{t}\right)=1 .
$$

In other words, if we think of 0 and $\infty$ to be a single point, the 'reverse Markov process' converges to this point almost surely. 
Proof. Equivalently, we show that

$$
\operatorname{Pr}\left(y_{t} \in\left[z_{1}, z_{2}\right], \text { i.o. }\right)=0 .
$$

By the Borel-Cantelli lemma, it is sufficient to show that

$$
\sum_{t=-1}^{-\infty} \operatorname{Pr}\left(y_{t} \in\left[z_{1}, z_{2}\right]\right)<\infty .
$$

We show this by contradiction; suppose

$$
\sum_{t=-1}^{-\infty} \operatorname{Pr}\left(y_{t} \in\left[z_{1}, z_{2}\right]\right)=\infty
$$

Let $A_{t}$ be the event $\left\{y_{t} \in\left[z_{1}, z_{2}\right], y_{s} \in\left(0, z_{1}\right) \cup\left(z_{2}, \infty\right), \forall s \leq t-1\right\}$. Since $\operatorname{Pr}\left(A_{t}\right) \geq \operatorname{Pr}\left(y_{t} \in\right.$ $\left.\left[z_{1}, z_{2}\right]\right) \pi$,

$$
\begin{aligned}
\sum_{t=-1}^{-\infty} \operatorname{Pr}\left(A_{t}\right) & \geq \sum_{\substack{t=-1\\
}}^{-\infty} \operatorname{Pr}\left(y_{t} \in\left[z_{1}, z_{2}\right]\right) \pi \\
& \infty
\end{aligned}
$$

This is a contradiction to the fact that $\left\{A_{t}\right\}_{t=-1}^{-\infty}$ is a sequence of disjoint sets.

Proposition 3 Suppose $\varepsilon$ is an unbounded shock, then under Assumptions 1-5, the invariant distribution is unique.

Proof. By contradiction, suppose there are two invariant distributions, with distribution functions $F_{1}, F_{2}$. For any $y_{0}>0$ :

$$
\begin{aligned}
& F_{1}\left(y_{0}\right)=\int F_{1}\left(h^{-1}\left(y_{0} / \varepsilon\right)\right) \mu(d \varepsilon), \\
& F_{2}\left(y_{0}\right)=\int F_{2}\left(h^{-1}\left(y_{0} / \varepsilon\right)\right) \mu(d \varepsilon) .
\end{aligned}
$$

If $\left\{\varepsilon_{0}\right\}_{t=0}^{-\infty}$ is a sequence of i.i.d. random variables with distribution $\mu$, and $\left\{y_{t}\right\}_{t=0}^{-\infty}$ is the 'reverse Markov process',

$$
\begin{aligned}
F_{1}\left(y_{0}\right) & =\int F_{1}\left(y_{-1}\right) \mu\left(d \varepsilon_{0}\right) \\
& =\int F_{1}\left(y_{-2}\right) \mu\left(d \varepsilon_{-1}\right) \mu\left(d \varepsilon_{0}\right) \\
& =\int F_{1}\left(y_{-3}\right) \mu\left(d \varepsilon_{-2}\right) \mu\left(d \varepsilon_{-1}\right) \mu\left(d \varepsilon_{0}\right) \\
& =\int F_{1}\left(y_{-k}\right) \mu\left(d \varepsilon_{-k}\right) \cdots \mu\left(d \varepsilon_{0}\right) .
\end{aligned}
$$


Since $\lim _{t \rightarrow(-\infty)} y_{t}=0($ or $\infty)$ a.s., by the dominated convergence theorem,

$$
\begin{aligned}
F_{1}\left(y_{0}\right)-F_{2}\left(y_{0}\right) & =\int\left(F_{1}-F_{2}\right)\left(y_{-k}\right) \mu\left(d \varepsilon_{-k}\right) \cdots \mu\left(d \varepsilon_{0}\right) \\
& =\lim _{(-k) \rightarrow(-\infty)} \int\left(F_{1}-F_{2}\right)\left(y_{-k}\right) \mu\left(d \varepsilon_{-k}\right) \cdots \mu\left(d \varepsilon_{0}\right) \\
& =\int\left[\lim _{t \rightarrow(-\infty)}\left(F_{1}-F_{2}\right)\left(y_{t}\right)\right]\left[\otimes_{t=0}^{-\infty} \mu\left(d \varepsilon_{t}\right)\right] \\
& =\int 0\left[\otimes_{t=0}^{-\infty} \mu\left(d \varepsilon_{t}\right)\right] \\
& =0 .
\end{aligned}
$$

Therefore $F_{1}\left(y_{0}\right)-F_{2}\left(y_{0}\right)=0$, for all $y_{0}>0$. The distribution is unique.

The above proposition and Brock and Mirman (1972) give a complete solution to uniqueness with general shocks.

\section{Concluding comments}

- Stachurski (2002) and Nishimura and Stachurski (2005) studied a stronger notion of stability when they focused on shocks with densities. They showed that the distribution will converge in total variation norm under certain conditions. However, in general, it is impossible to prove this 'strong convergence' when we allow the shock to have discrete values. This can be made clear when we look at the deterministic growth model. It is well known that when $\varepsilon$ is a constant, there exists a steady state $y^{*}>0$, such that if $y_{0}<y^{*}$, then $y_{0}<y_{t}<y_{t+1}<y^{*}$ for every $t$. It is easy to show that the unique invariant distribution is $\delta_{y^{*}}$. The importance of this example is to suggest the appropriate topology for convergence results in general. The total variation norm does not work in this case, since if $y<y^{*}$, then $\left\|T^{*} \delta_{y}-\delta_{y}\right\|=\left\|\delta_{h(y) \varepsilon}-\delta_{y}\right\|=2$. This is why we use weak convergence for stability analysis.

- Although the idea behind our method is fairly general, it might be difficult to apply when studying other types of dynamic systems with non-compact state space. This is mainly because of the difficulty in constructing the boundary distributions to start with. In this paper, this is achieved by the careful use of Euler equations near 
boundaries. In order to apply this method to other dynamic systems, equal care in understanding the behavior near the boundaries of those systems is required.

\section{Acknowledgements}

I thank V.V. Chari and Larry Jones for their continous encourage and support. I am very grateful to Andrew Mclennan for the numerous disscussions with him about this paper. I also thank him for his help with the revision of this manuscript. All remaining errors are mine.

\section{References}

C.D. Aliprantis and K.C. Border. Infinite Dimensional Analysis: A Hitchhiker's Guide. Springer Verlag, 2nd revised edition edition, September 1999.

W.A. Brock and L. Mirman. Optimal economic growth and uncertainty: the discounted case. Journal of Economic Theory, 4:479-513, 1972.

D. Cass. Optimum growth in an aggregative model of capital accumulation. Review of Economic Studies, 32:233-240, 1965.

D. Duffie, J. Geanakoplos, A. Mas-Colell, and A. McLennan. Stationary markov equilibria. Econometrica, 62(4):745-781, 1994.

C.A. Futia. Invariant distributions and the limiting behavior of markovian economic models. Econometrica, 50:377-408, 1982.

H.A. Hopenhayn and E.C. Prescott. Stochastic monotonicity and stationary distributions for dynamic economies. Econometrica, 60(6):1387-1406, 1992.

T. Koopmans. On the concept of optimal economic growth. Pontificiae Academiae Scientiarum Scripta Varia, 28:225-300, 1965.

A. Lasota. Invariant principle for discrete time dynamical systems. Univ. Iagel. Acta Math., 31:111-127, 1994. 
S.P. Meyn and R.L. Tweedie. Markov Chains and Stochastic Stability. Springer, London, 1993.

L.J. Mirman. On the existence of steady-state measures for one-sector growth models with uncertain technology. International Economic Review, 13:271-286, 1972.

L.J. Mirman and I. Zilcha. On optimal growth under uncertainty. Journal of Economic Theory, 11:329-339, 1975.

K. Nishimura and J. Stachurski. Stability of stochastic optimal growth models: a new approach. Journal of Economic Theory, 122:100-118, 2005.

F.P. Ramsey. A mathematical theory of saving. Economic Journal, 38:543-559, 1928.

J. Stachurski. Stochastic optimal growth with unbounded shock. Journal of Economic Theory, 106:40-65, 2002.

N.L. Stokey, R.E. Lucas, and E.C. Prescott. Recursive Methods in Economic Dynamics. Harvard University Press, Cambridge MA, 1989. 